\title{
Power law behavior of Elementary Cellular Automata
}

\author{
Jorge A. Laval ${ }^{1, *}$ \\ ${ }^{1}$ School of Civil and Environmental Engineering, Georgia Institute of Technology
}

(Dated: Version June 26, 2021)

\begin{abstract}
This paper shows that the percolation clusters from elementary cellular automata 30, 45, 60, 86, 99, 105, 129, 150, 153, 169, 182, 183, 184, 195 and 225 exhibit strong power law behavior, either under random initial conditions, a single occupied cell, or both. Most of the tail exponents are less than unity, implying diverging means and variances of cluster sizes. The analysis presented here is admittedly coarse in an effort to expedite its dissemination.
\end{abstract}

Keywords: Elementary Cellular automata, Power laws, fractals, criticality

\section{INTRODUCTION}

This short paper shows that the percolation clusters from 15 elementary cellular automata [15] (ECA) exhibit strong power law behavior. It appears that this result has not been noted in the literature and could have important implications in a wide variety of subjects, especially since ECAs are the simplest rules to produce complex and computational irreducible behaviors. The analysis presented here is therefore admittedly coarse in an effort to expedite its dissemination.

A growing list of phenomena in all areas of science exhibit power law behavior, which has puzzled scientists for generations: from the floods of the Nile River [7] to the population of cities [11], earthquakes, allometric scaling in mammals [14], word frequencies [17], reference links on the web [12], financial returns [10], the size (number of employees, sales, assets) of companies [9, 13], turbulent flows and many others [4].

Significant progress has been made to advance conjectures to explain these phenomena borrowing from the theory of phrase transitions, critical phenomena and selforganized criticality $[2,6,8]$ : at the critical point the characteristic length scale of the system, such as the mean cluster size in percolation models, diverges to infinity, which renders the system scale-free and thus scale invariant, the hallmark of power laws. But a general theory is still lacking and there are still many open questions [1].

\section{METHOD}

All 256 ECAs rules were evolved for $T=5,000$ time periods starting with an initial row of $L=10,000$ cells, each with a probability $p$ of being occupied. The relationship $L=2 T$ is used here to minimize the effect of the periodic boundary conditions. The tail distribution of cluster sizes were plotted on a log-log plot so that the slope of the linear segment (if any) gives an idea of the

\footnotetext{
* jorge.laval@ce.gatech.edu
}

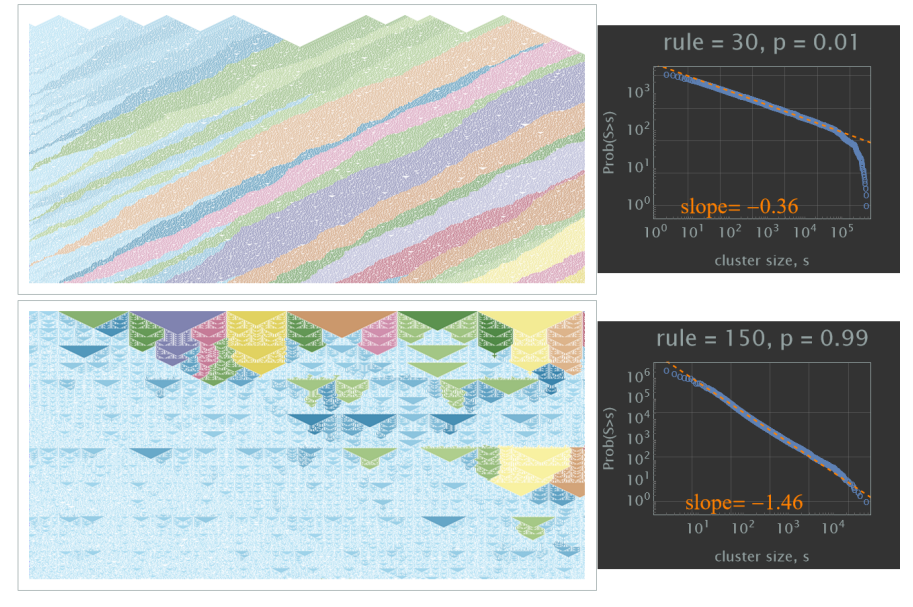

Figure 1. Log-log probability plots and sample spatiotemporal realization for rules 30 and 150 .

exponent of the power law; see Fig. 1. The appendix presents similar figures for all rules identified here.

The exponents reported below are only approximate, and were estimated using piecewise linear regression in order to account for finite-size effects. Only linear relationships over 4 orders of magnitude or more were considered here.

\section{RESULTS}

We found 4 types of power law behavior depending on their sensitivity to the occupational probability. In all cases the power law relationship is very strong at all orders of magnitude, with cutoffs that increase with the grid size, suggesting the validity of these relationships at all scales.

1. Class 1: 30, 45, 60. Robust exponent $0.36 \pm .01$ independent of the occupation probability.

2. Class 2: 182, 183. Strong power law relationship at all occupation probabilities, but with exponent dependent on the occupation probability in the extremes $p \rightarrow 0, p \rightarrow 1$. 


\begin{tabular}{|cccc|c|c|c|c|}
\hline \multirow{2}{*}{$\begin{array}{c}\text { PL } \\
\text { class }\end{array}$} & Rule Wolfram & Equiv. & \multicolumn{4}{|c|}{ Tail exponent } \\
\cline { 5 - 9 } & & class & rules & $p=.5$ & .01 & .99 & single \\
\hline \hline 1 & 30 & III & 86,135 & 0.36 & 0.36 & 0.36 & 0.22 \\
1 & 45 & III & $75,89,101$ & 0.36 & 0.36 & 0.36 & \\
1 & 60 & III & $102,153,195$ & 0.36 & 0.36 & 0.36 & \\
\hline 2 & 182 & III & 146 & 0.71 & 0.6 & 0.6 & \\
2 & 183 & III & 18 & 0.71 & 0.6 & 0.6 & \\
\hline 3 & 129 & III & 126 & & 1.75 & 1.75 & 0.75 \\
3 & 153 & III & $60,102,195$ & & 1.15 & 1.15 & 0.78 \\
3 & 195 & III & $60,102,195$ & & 1.15 & 1.15 & 0.78 \\
\hline 4 & 99 & II & 57 & 0.71 & & & \\
4 & 105 & III & - & & & 0.9 & 0.65 \\
4 & 150 & III & - & & & 1.5 & 1.73 \\
4 & 184 & II & 226 & 0.5 & & & \\
\hline
\end{tabular}

Table I. Summary of exponents for random initial conditions at 3 levels of the occupation probability, $p=.5, .01, .99$. The last column represents the single-occupied-cell initial condition. Empty table cells: no power law relationship.

3. Class 3: 129, 153, 195. Strong power law relationship only in the extremes $p \rightarrow 0, p \rightarrow 1$.

4. Class 4: 99, 105, 150, 184. Strong pIower law relationship at one particular occupation probability.

Table 1 summarizes the results. Note how that in most cases the equivalent rules $^{1}$. As can be seen from the appendix, classes 1 and 2 exhibit very intricate and complex patterns that tend to span the whole grid, while classes 3 and 4 produces cluster patterns reminiscent of the Sierpinski triangle which tend to stay localized.

To better understand the behavior in the limits $p \rightarrow$ $0, p \rightarrow 1$, we repeated the analysis for initial conditions given by a single occupied cell in a background of empty cells, and its complement, using $T=10,000$ time periods. Exponents are summarized below:
Rule: $\quad 30 \quad 86 * \quad 105 \quad 169 * 225^{*} \quad 129 \quad 153 \quad 195 \quad 150$

\begin{tabular}{lllllllllll}
\hline single 1: & 0.22 & 0.22 & 0.65 & 0.72 & 0.72 & 0.75 & 0.78 & 0.78 & 1.73
\end{tabular}

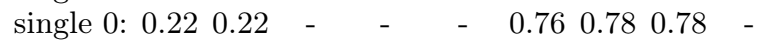

The missing entries mean that no cluster structures exist for that configuration. The $*$ indicates that the rule does not exhibit power law behavior under random initial conditions. Notice the sharp differences compared to the cases $p=.01, p=.99$ from Table 1 , possibly indicating a phase transition.

\section{DISCUSSION}

Power law behavior is used here in the strict sense of cluster sizes having a power law tail distribution. This is a necessary but not sufficient condition for criticality, which will have to be scrutinized further. Criticality was conjectured for rule $22[5,16]$ but no percolation analysis was performed. Here we did not find strong evidence of power law cluster behavior for rules 22 or its complement, 151. It has been reported that critical behavior in ECA arises with asynchronous updating, or by combining rules 22 and 4 [3], but here we have shown that it is possible that critical behavior is intrinsic to some of the rules identified here.

Notice that with the exception of rules 99 and 184, the rules identified here belong to Wolfram class III. Rules 90, 122, 126 in Wolfram class III are the only ones in this class not to exhibit power law behavior. Interestingly, none of Wolfram class IV were found to exhibit this behavior.

Recall that the exponents presented here are approximate and further analysis is warranted to refine these estimates. Power law classes 1 and 2 identified here appear very robust to changes in the grid size, and it is expected that future refinements will not alter the reported values very significantly. But class 3 exhibits the most variability when changing the grid size and should be studied more carefully.
[1] Aschwanden, M.J., Crosby, N.B., Dimitropoulou, M., Georgoulis, M.K., Hergarten, S., McAteer, J., Milovanov, A.V., Mineshige, S., Morales, L., Nishizuka, N., et al., 2016. 25 years of self-organized criticality: solar and astrophysics. Space Science Reviews 198, 47-166.

[2] Bak, P., Tang, C., Wiesenfeld, K., 1987. Self-organized criticality: an explanation of 1 /f noise. Phys. Rev. Lett $59,381$.

[3] Bhattacharyya, P., 1996. Critical phenomena in an one-

\footnotetext{
${ }^{1}$ Each rule is equivalent to three others: by interchanging black and white cells, by interchanging the left and right cells, and by performing both transformations.
}

dimensional probabilistic cellular automation. Physica A: Statistical Mechanics and its Applications 234, 427-434.

[4] Clauset, A., Shalizi, C.R., Newman, M.E., 2009. Powerlaw distributions in empirical data. SIAM review 51, 661-703.

[5] Eisele, M., 1991. Long-range correlations in chaotic cellular automata. Physica D: Nonlinear Phenomena 48, 295-310.

[6] Halperin, B., Hohenberg, P., 1969. Scaling laws for dynamic critical phenomena. Physical Review 177, 952.

[7] Hurst, H.E., 1951. Long-term storage capacity of reservoirs. Transactions of the American society of civil engineers 116, 770-799.

[8] Khaluf, Y., Ferrante, E., Simoens, P., Huepe, C., 2017. Scale invariance in natural and artificial collective sys- 
tems: a review. Journal of the royal society interface 14, 20170662.

[9] Kobayashi, Y., Takayasu, H., Havlin, S., Takayasu, M., 2021. Robust characterization of multidimensional scaling relations between size measures for business firms. Entropy 23, 168.

[10] Mandelbrot, B., Hudson, R.L., 2007. The Misbehavior of Markets: A fractal view of financial turbulence. Basic books.

[11] Mori, T., Smith, T.E., Hsu, W.T., 2020. Common power laws for cities and spatial fractal structures. Proceedings of the National Academy of Sciences 117, 6469-6475.

[12] Newman, M.E., 2005. Power laws, pareto distributions and zipf's law. Contemporary physics 46, 323-351.

[13] Watanabe, H., Takayasu, H., Takayasu, M., 2013. Relations between allometric scalings and fluctuations in complex systems: The case of japanese firms. Physica A: Statistical Mechanics and its Applications 392, 741-756.

[14] West, G.B., Brown, J.H., Enquist, B.J., 1997. A general model for the origin of allometric scaling laws in biology. Science 276, 122-126.

[15] Wolfram, S., 1984. Cellular automata as models of complexity. Nature 311, 419.

[16] Zabolitzky, J.G., 1988. Critical properties of rule 22 elementary cellular automata. Journal of statistical physics 50, 1255-1262.

[17] Zipf, G., 1949. Human behaviour and the principle of least effort. 


\section{Appendix A: Log-log probability plots and spatiotemporal realizations}

This visual appendix includes the log-log probability plots along with a sample spatiotemporal realization for all the rules discussed here under random initial conditions for $p=.01, .5, .99$. Starting with the original configuration $L=$ $10,000, T=5,000$ in the next section, the following sections show similar plots on progressively smaller configurations, always with $L=2 T$. In this way, both the sensitivity of the exponents to the grid size and the self-similarities can be appreciated.

1. $L=10,000$
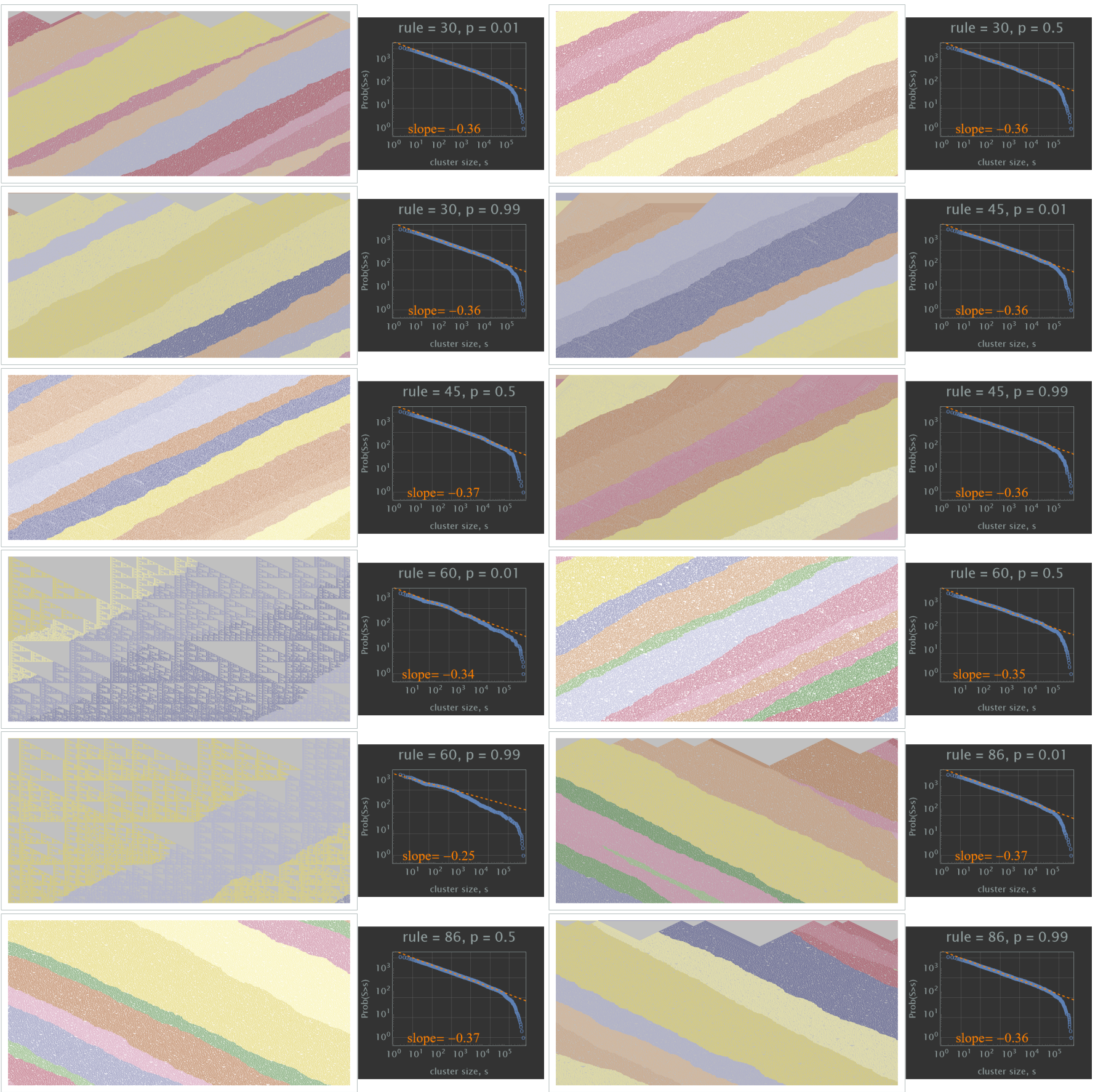

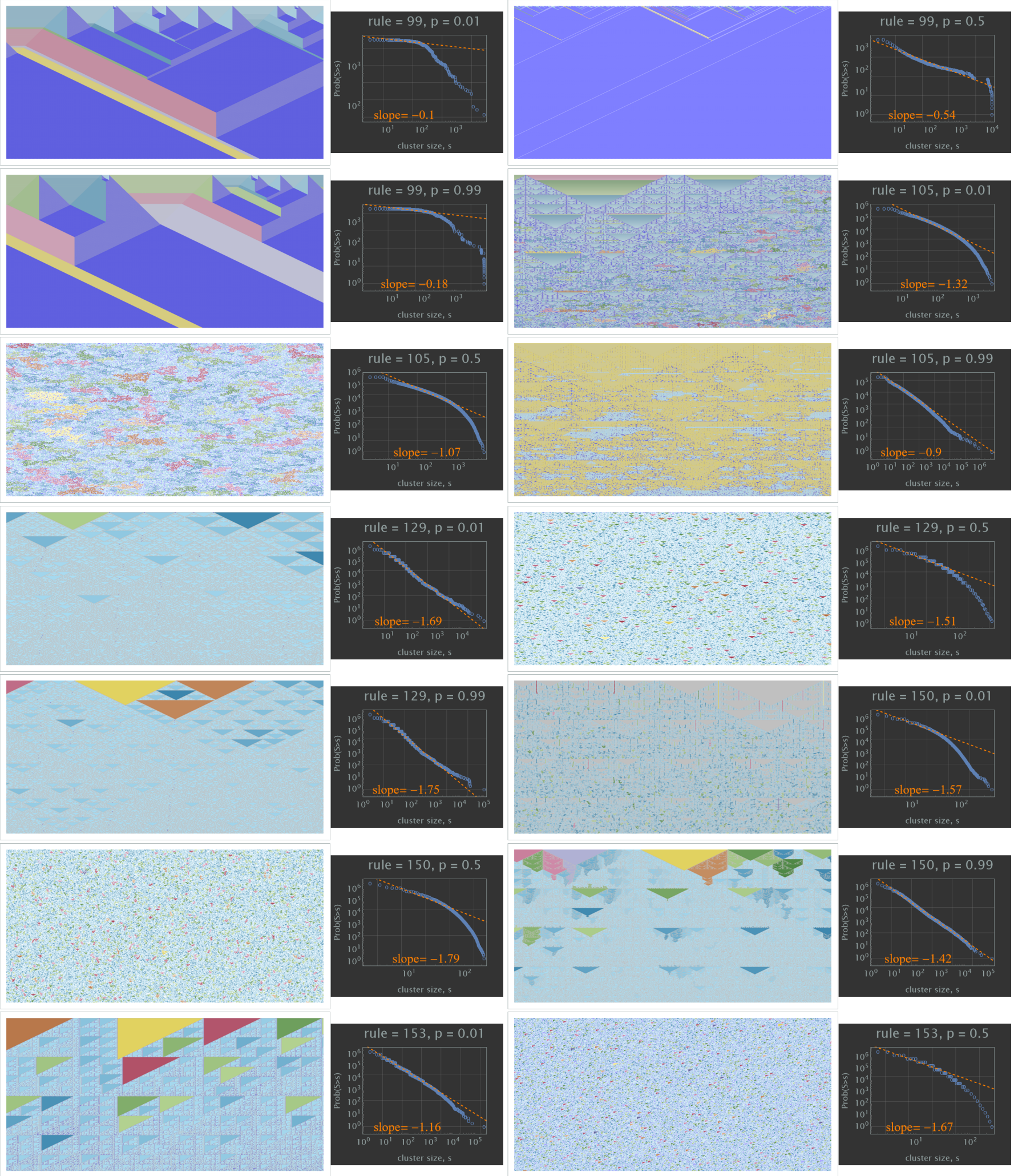

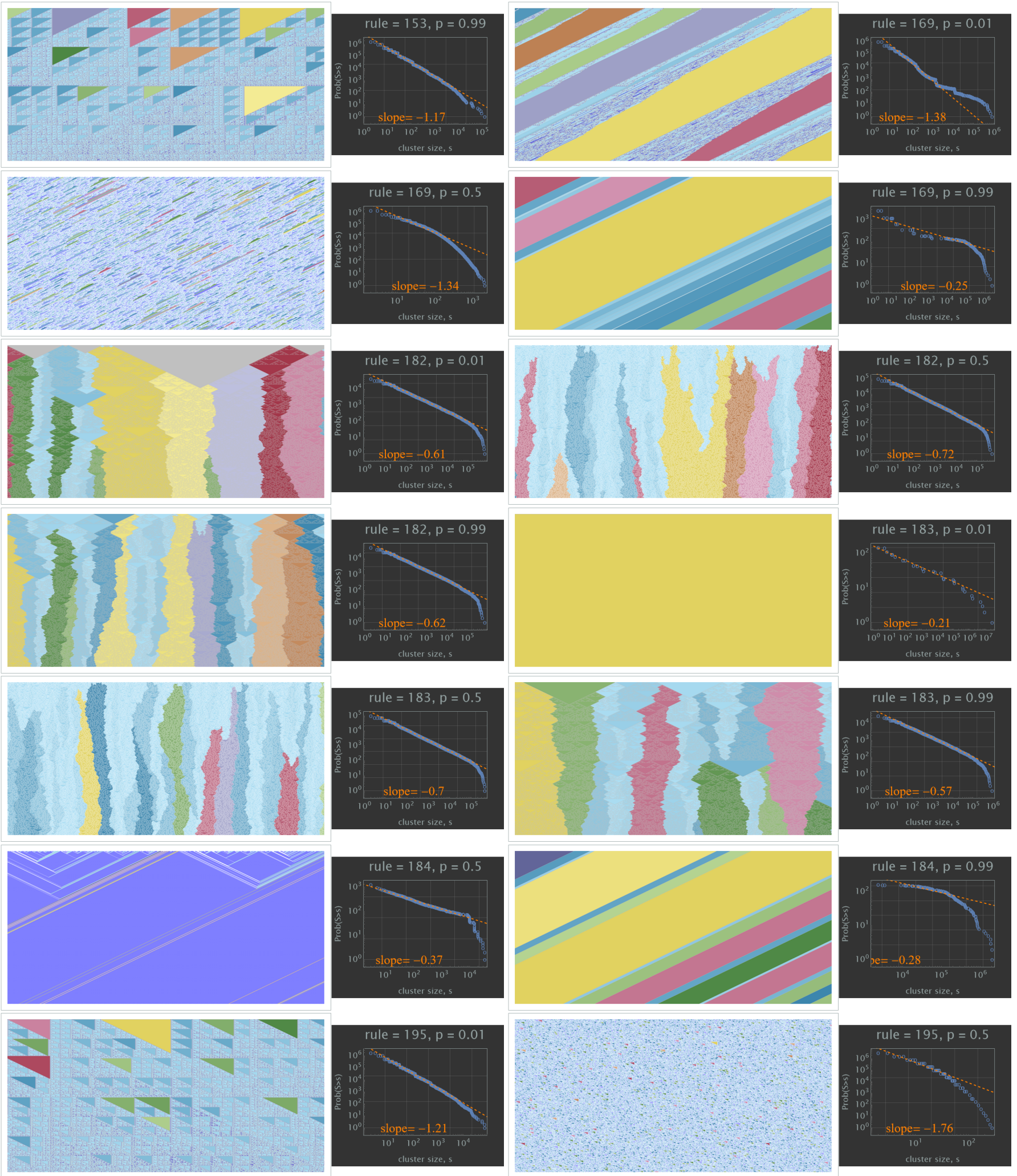

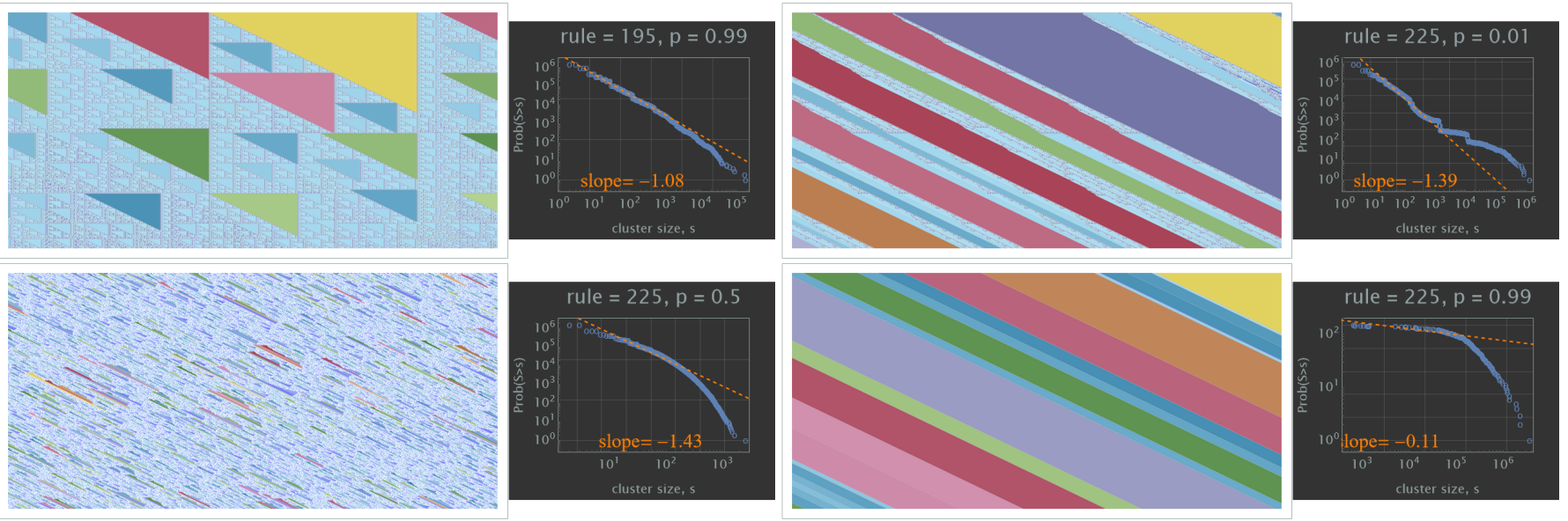

2. $L=400$
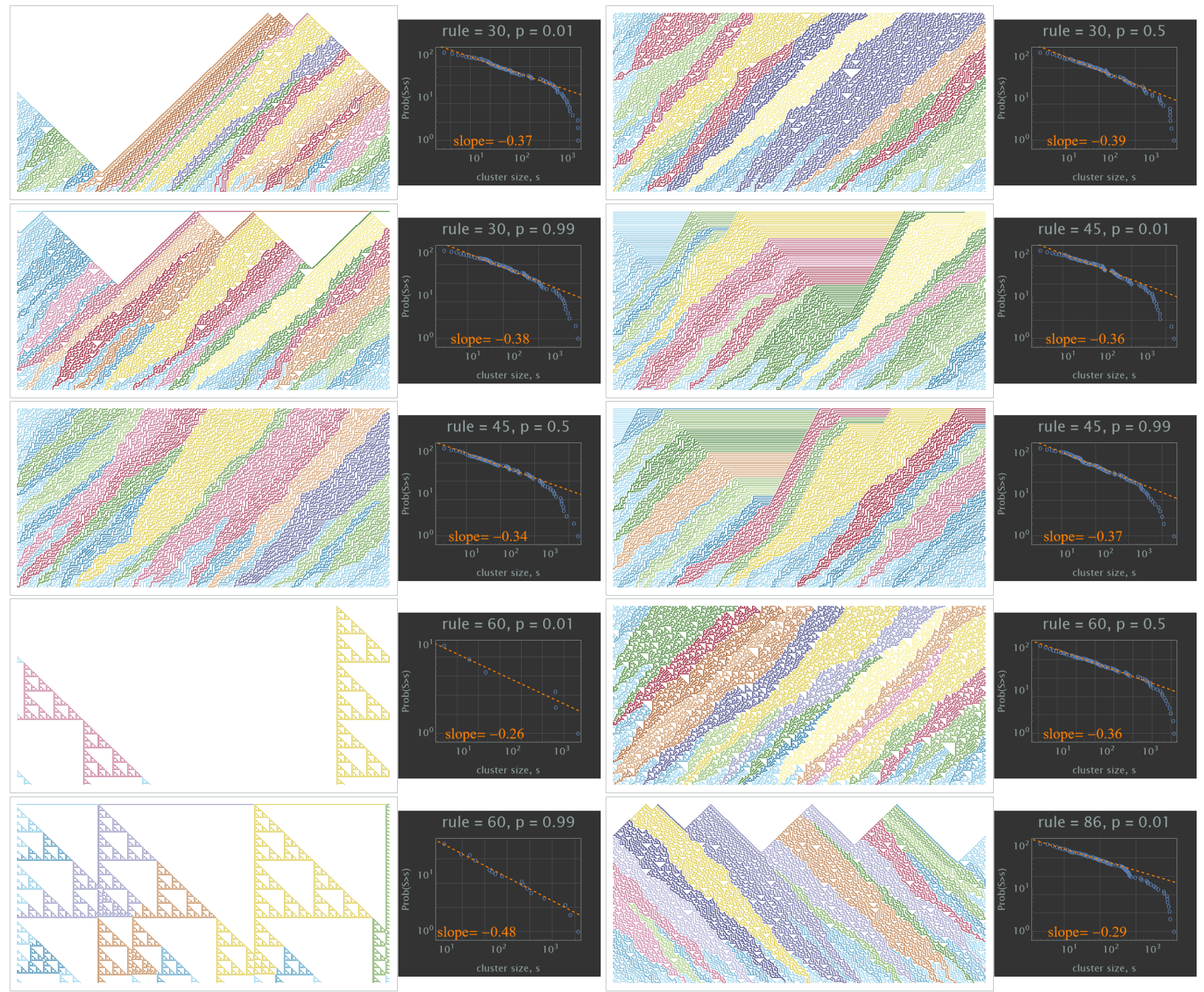

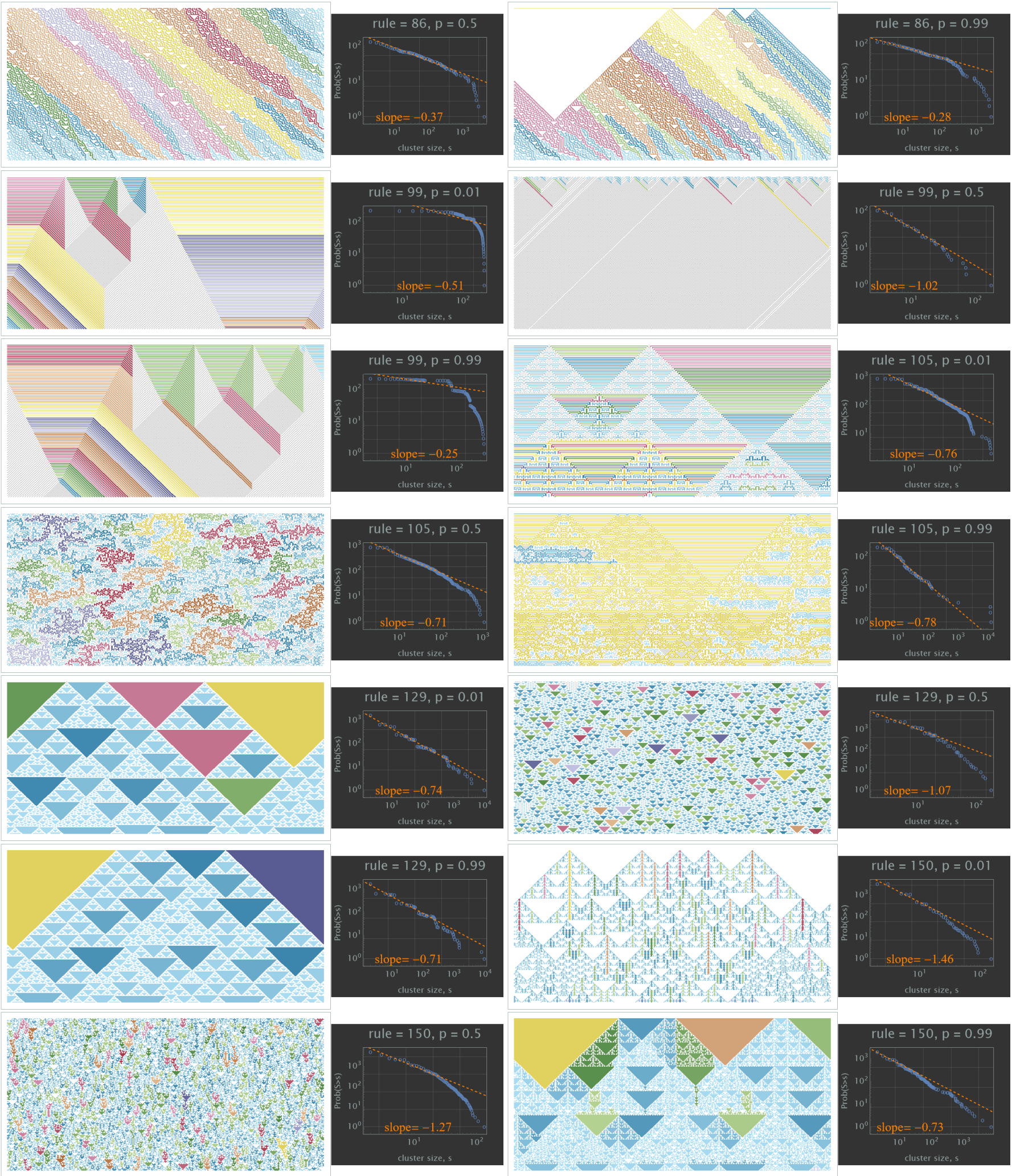

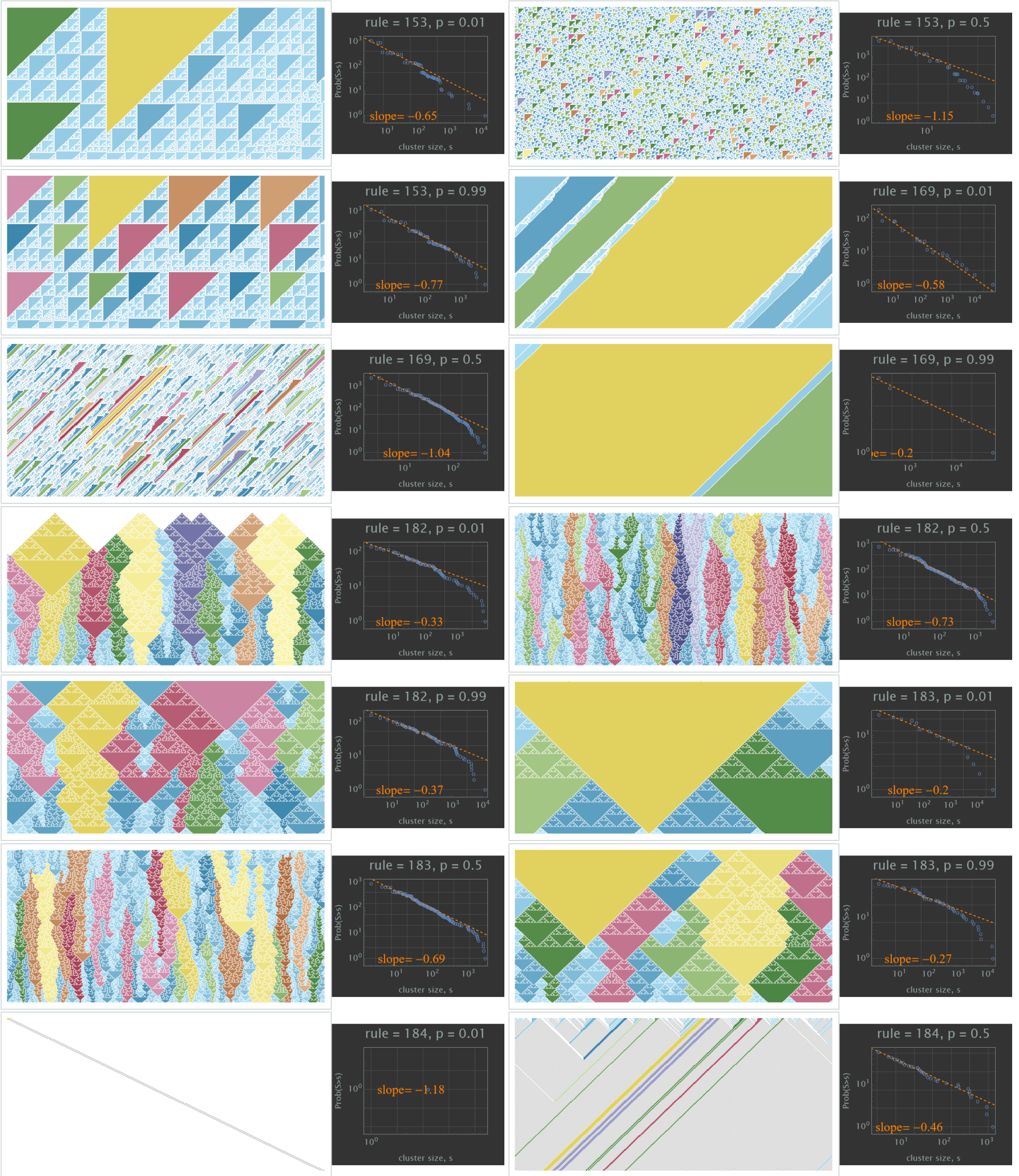

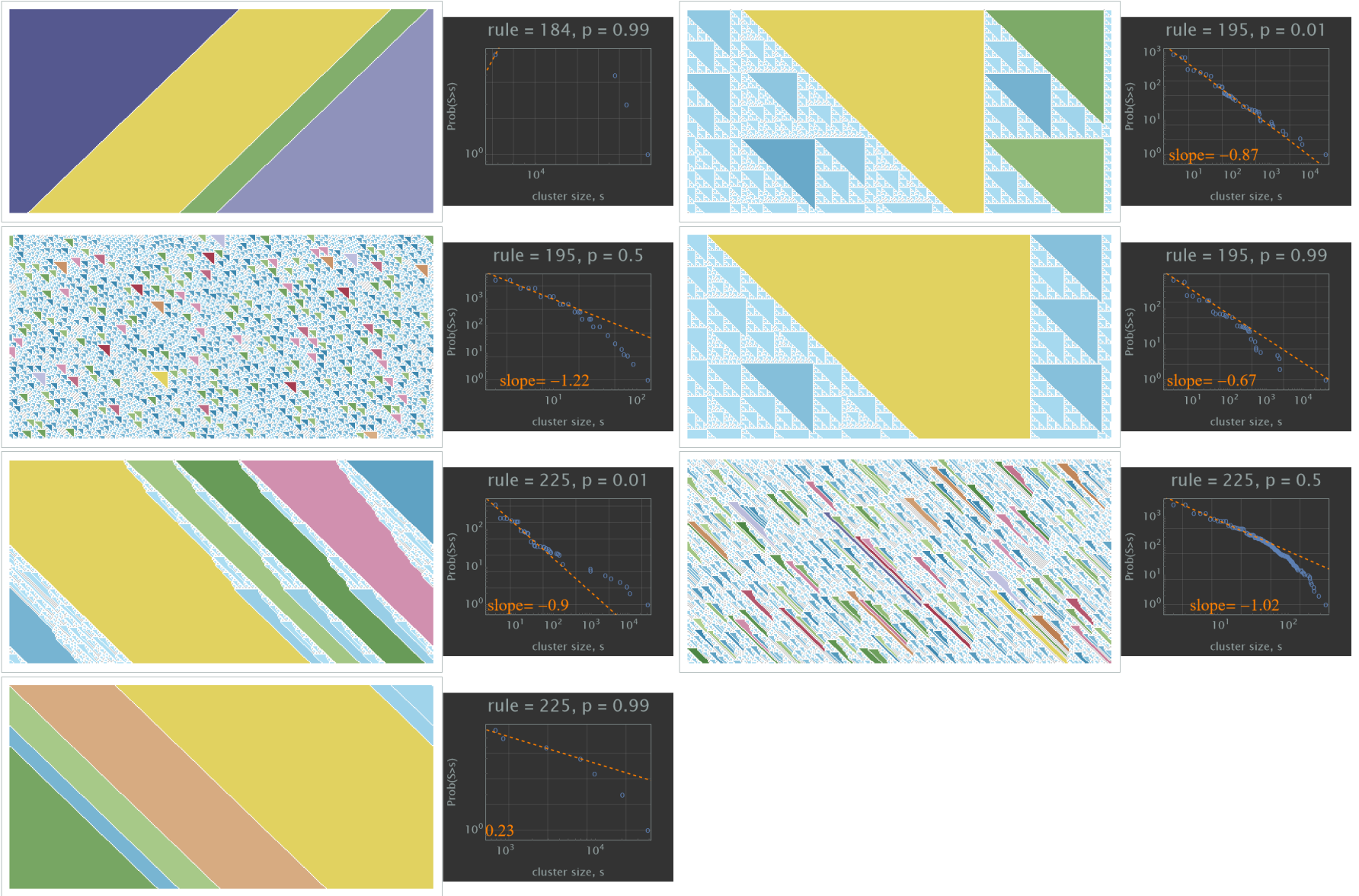

3. $L=100$
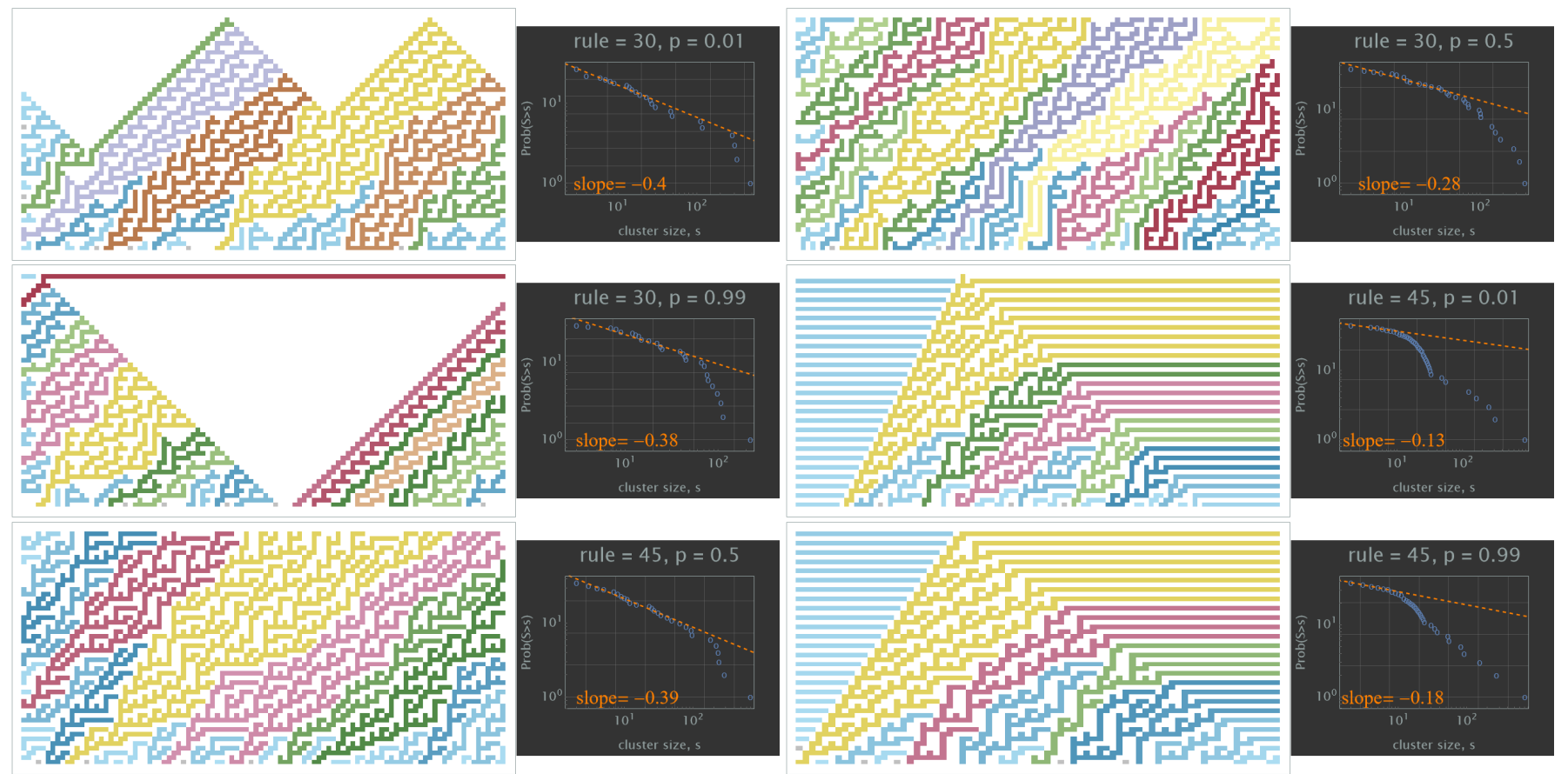


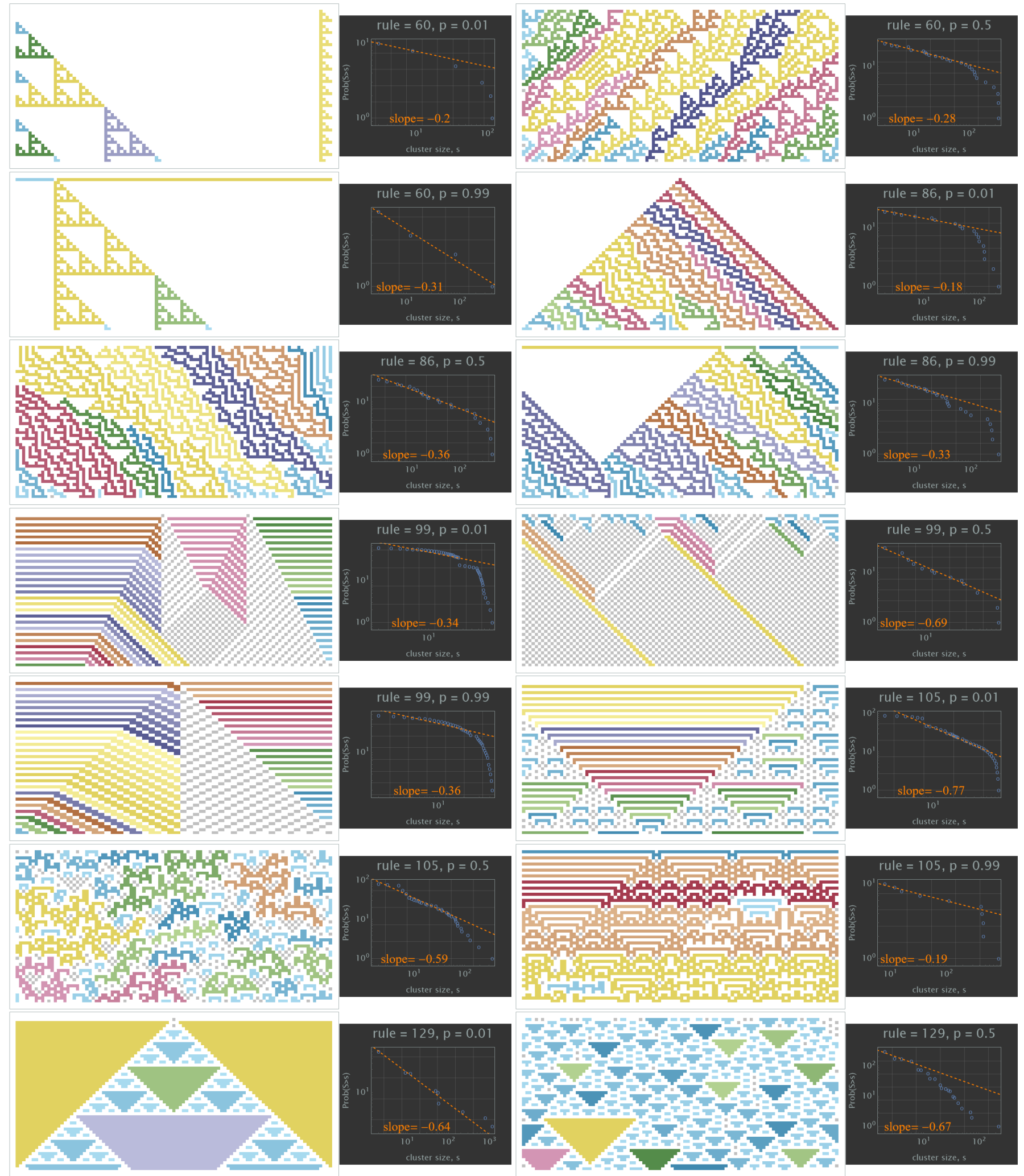




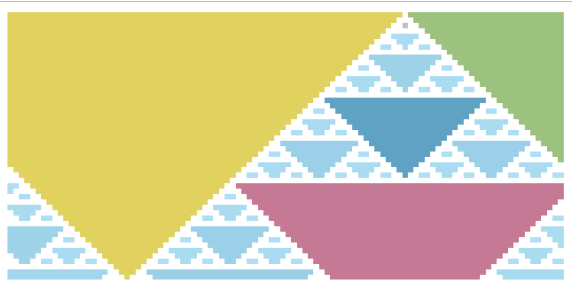

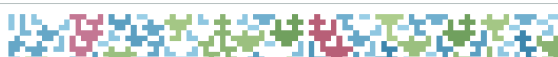

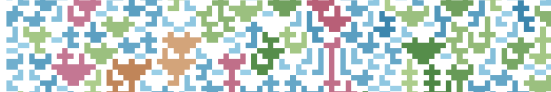

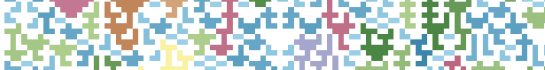
frity
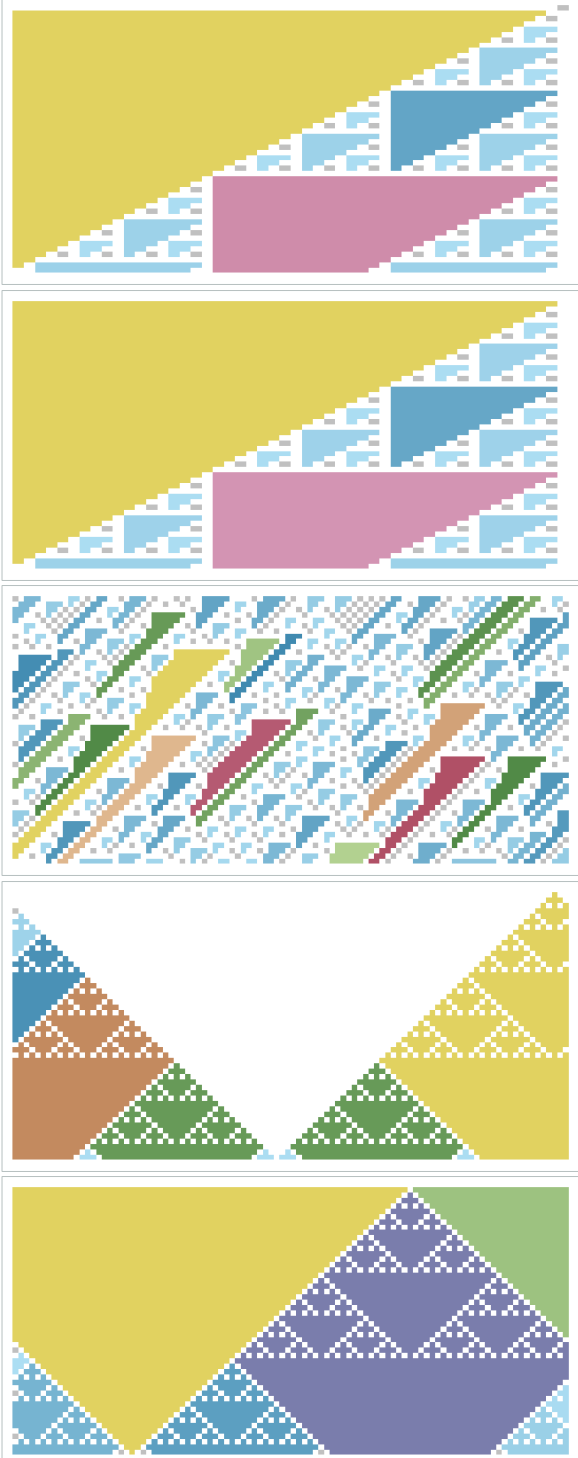
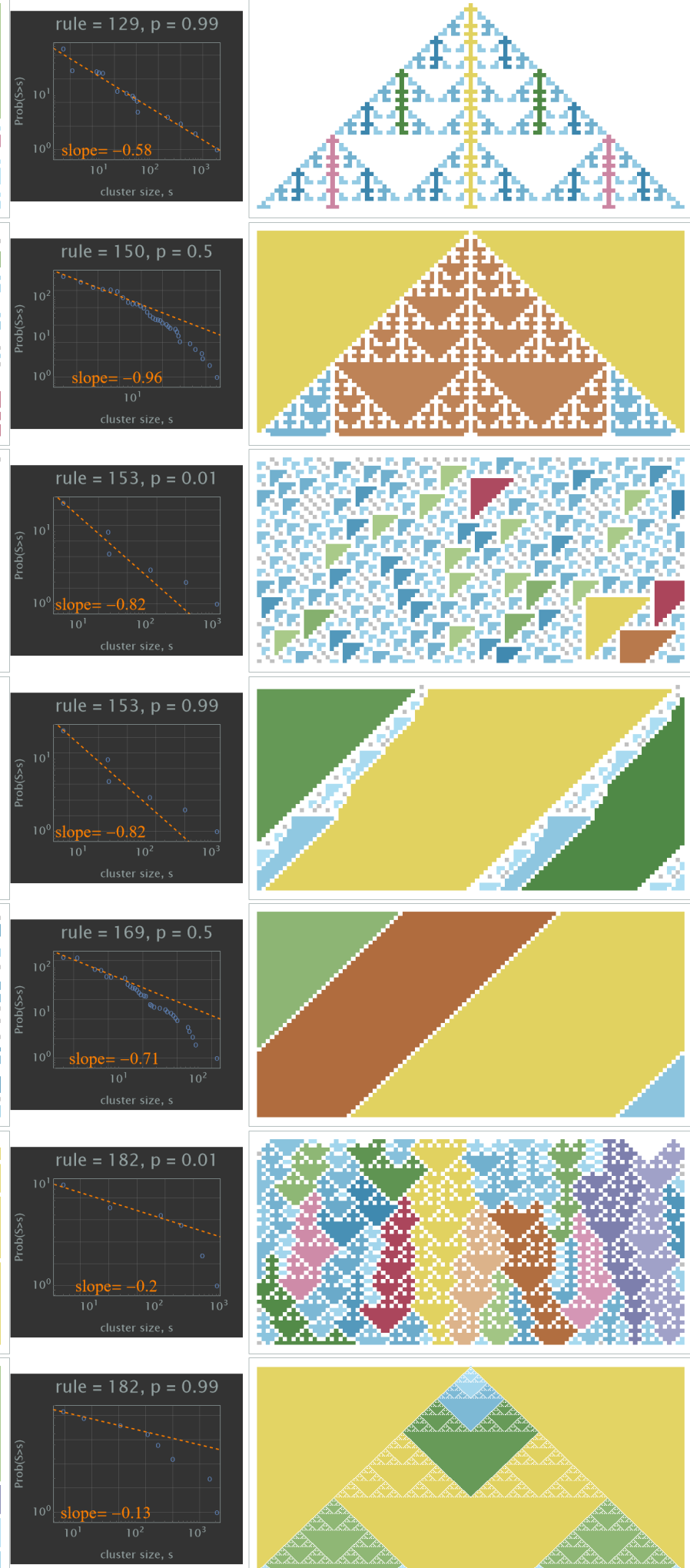
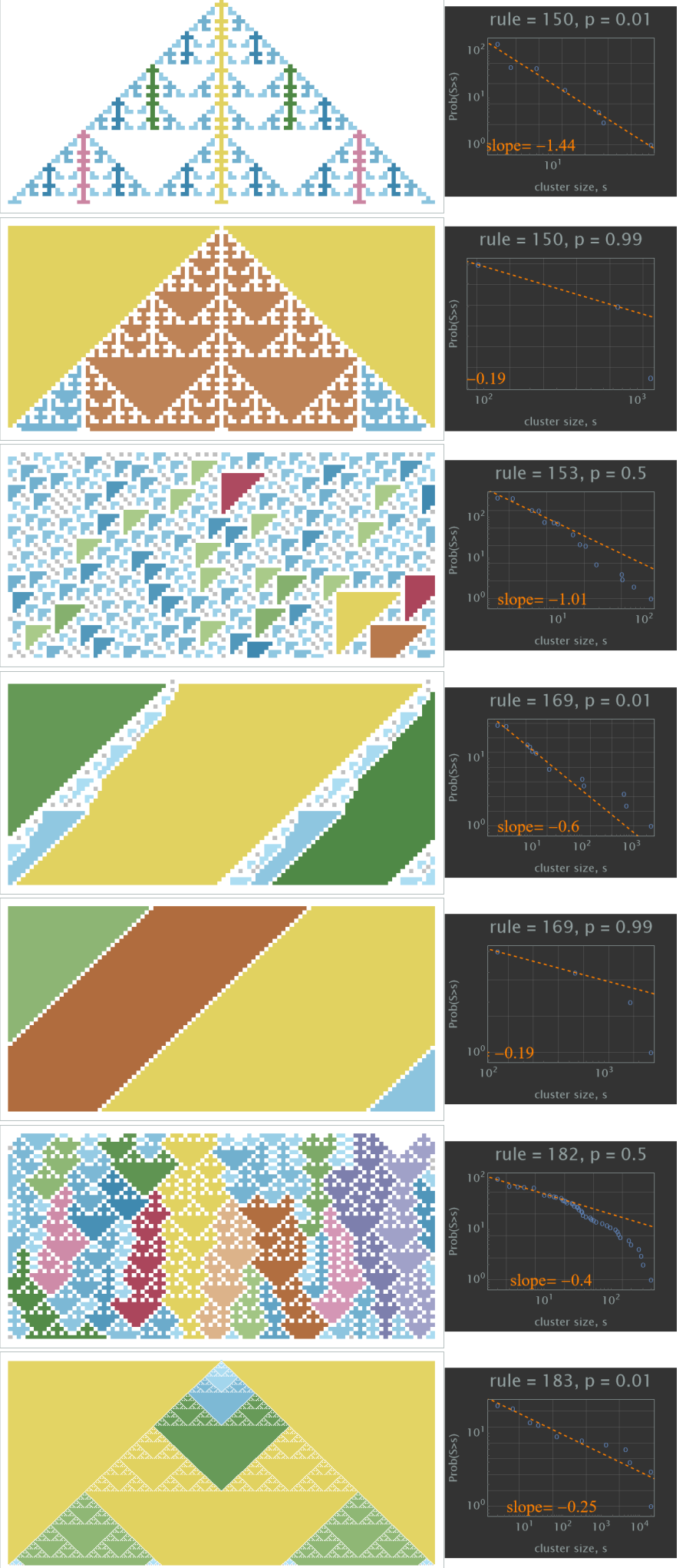

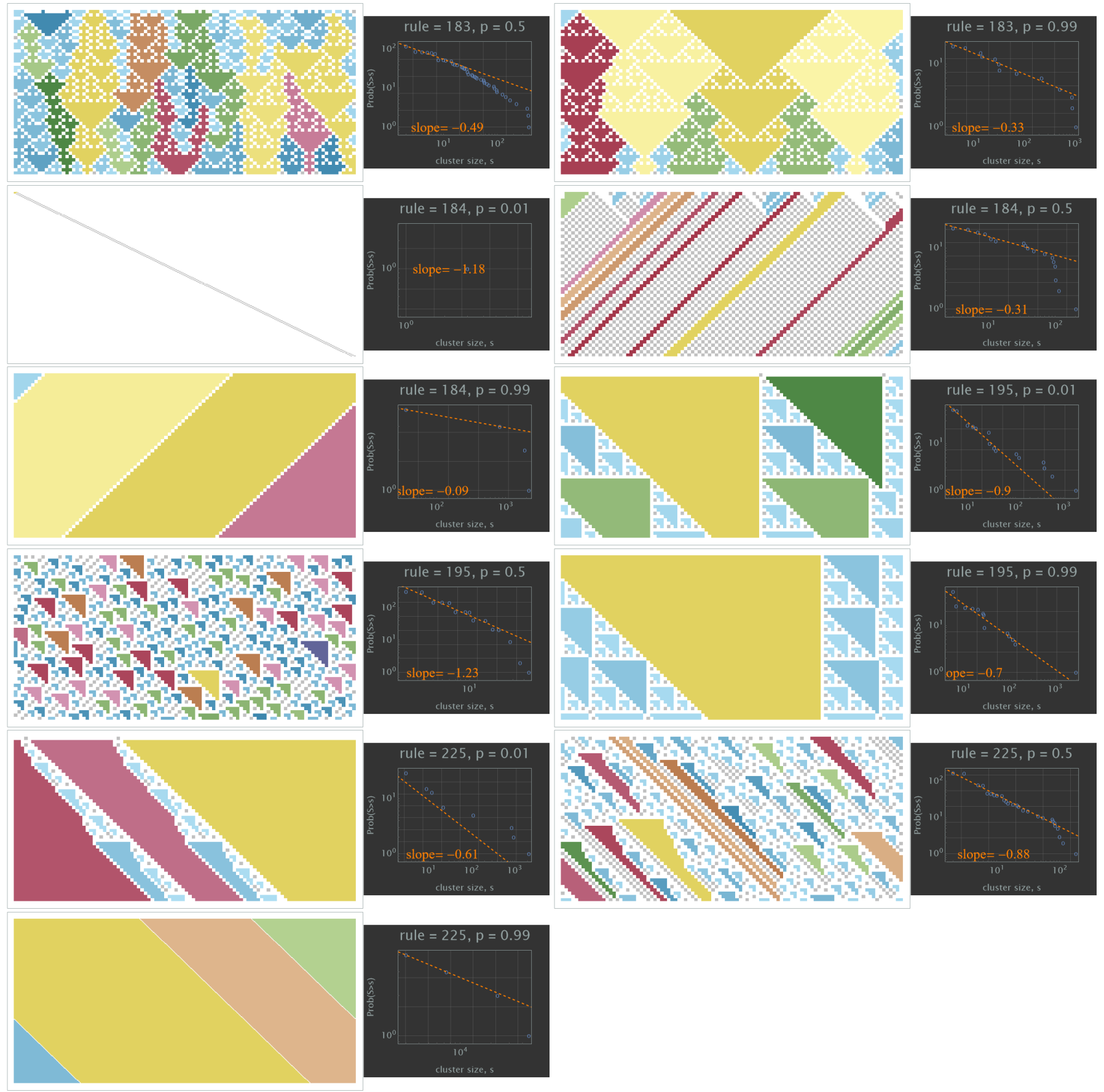